\title{
Human Reliability Implementation Guide
}

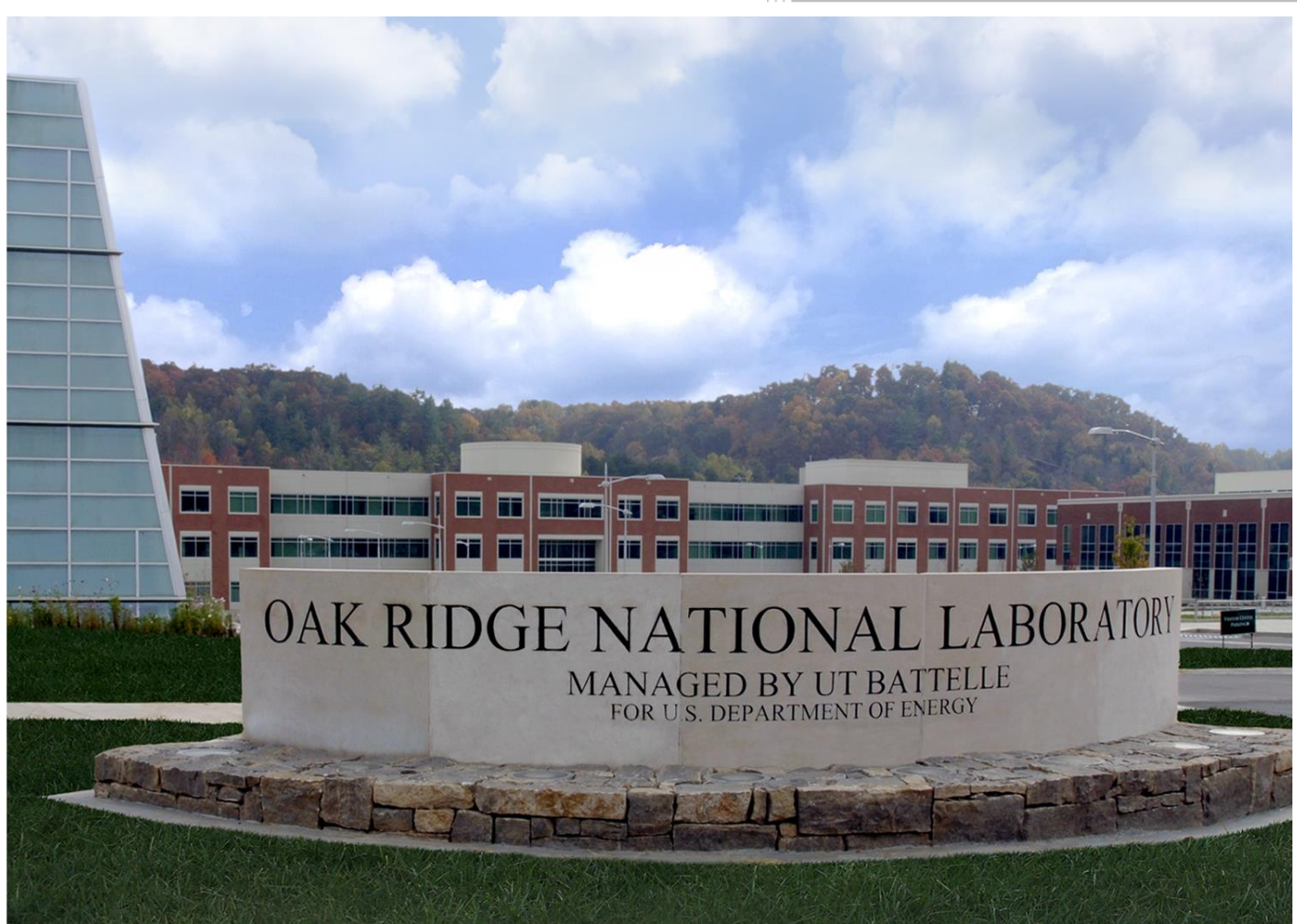

Approved for public release distribution is unlimited
Cameron W. Coates

Gerhard R. Eisele

August 2014 


\section{DOCUMENT AVAILABILITY}

Reports produced after January 1, 1996, are generally available free via US Department of Energy (DOE) SciTech Connect.

Website http://www.osti.gov/scitech/

Reports produced before January 1, 1996, may be purchased by members of the public from the following source:

National Technical Information Service

5285 Port Royal Road

Springfield, VA 22161

Telephone 703-605-6000 (1-800-553-6847)

TDD 703-487-4639

Fax 703-605-6900

E-mail info@ntis.gov

Website http://www.ntis.gov/help/ordermethods.aspx

Reports are available to DOE employees, DOE contractors, Energy Technology Data Exchange representatives, and International Nuclear Information System representatives from the following source:

Office of Scientific and Technical Information

PO Box 62

Oak Ridge, TN 37831

Telephone 865-576-8401

Fax 865-576-5728

E-mail reports@osti.gov

Website http://www.osti.gov/contact.html

This report was prepared as an account of work sponsored by an agency of the United States Government. Neither the United States Government nor any agency thereof, nor any of their employees, makes any warranty, express or implied, or assumes any legal liability or responsibility for the accuracy, completeness, or usefulness of any information, apparatus, product, or process disclosed, or represents that its use would not infringe privately owned rights. Reference herein to any specific commercial product, process, or service by trade name, trademark, manufacturer, or otherwise, does not necessarily constitute or imply its endorsement, recommendation, or favoring by the United States Government or any agency thereof. The views and opinions of authors expressed herein do not necessarily state or reflect those of the United States Government or any agency thereof. 
Nuclear Security and Isotope Technology Division

\title{
HUMAN RELIABILITY IMPLEMENTATION GUIDE
}

\author{
Cameron W. Coates \\ Gerhard R. Eisele
}

Date Published: August 2014

\author{
Prepared by \\ OAK RIDGE NATIONAL LABORATORY \\ Oak Ridge, Tennessee 37831-6283 \\ managed by \\ UT-BATTELLE, LLC \\ for the \\ US DEPARTMENT OF ENERGY \\ under contract DE-AC05-00OR22725
}




\section{CONTENTS}

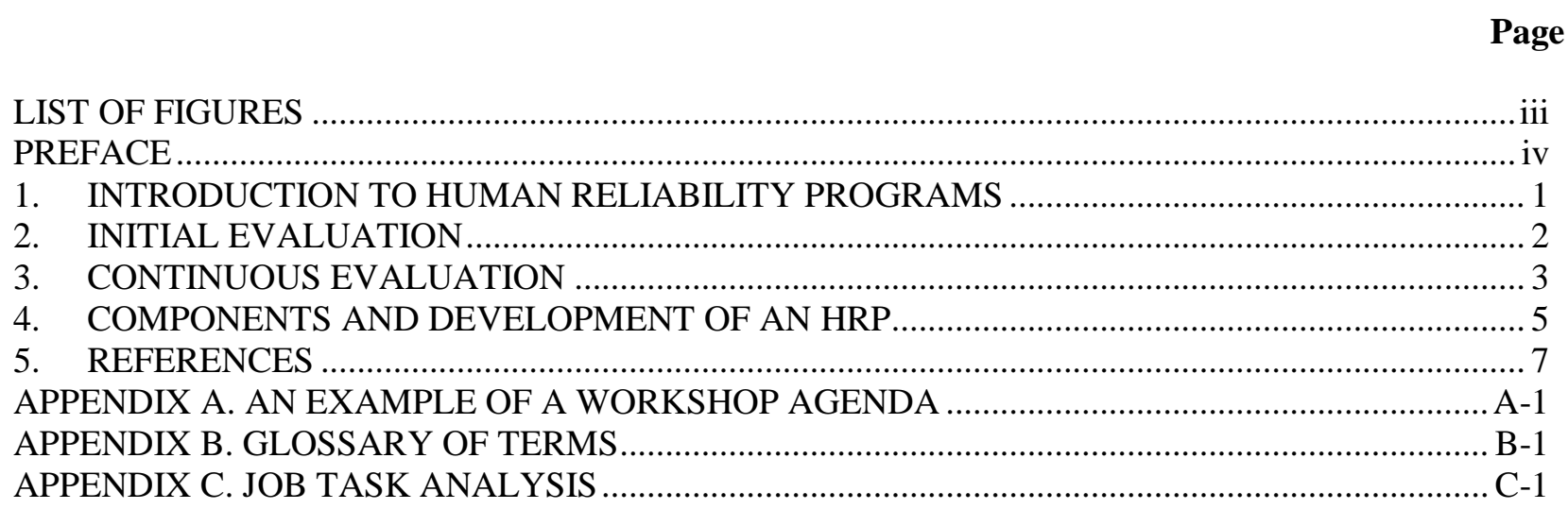

\section{LIST OF FIGURES}

Figure

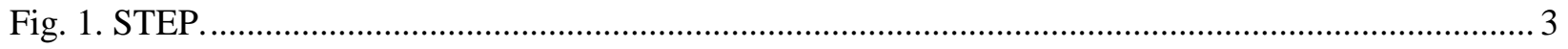




\section{PREFACE}

This implementation guide describes the process by which a Human Reliability Program (HRP) is established. It is intended to provide the reader with information regarding the importance of such a program and its various program elements. 


\section{INTRODUCTION TO HUMAN RELIABILITY PROGRAMS}

\section{What is a Human Reliability Program?}

A Human Reliability Program (HRP) is a "security and safety reliability program designed to ensure that individuals who occupy positions with access to certain nuclear materials, facilities, and programs meet the highest standards of reliability (an individual's ability to adhere to security and safety rules and regulations), trustworthiness (confidence in an individual based on his/her character) as well as physical and mental suitability." An HRP, also known as a Personnel Reliability Program (PRP) or a Structured Trusted Employee Program (STEP), ${ }^{2}$ recognizes the importance of selecting and retaining reliable, trustworthy, and suitable individuals to maintain secure and safe facilities.

\section{Why should an organization develop an HRP?}

The establishment of an HRP is one way to help mitigate potential insider risk, retain valued and trusted employees in critical/sensitive positions, and ensure employees meet the highest standards of reliability and trustworthiness. Each organization and its nuclear facilities have material and information deemed essential to its national security. An HRP can minimize the potential for infrastructure sabotage or the release of sensitive information. Safety and security systems can mitigate the risk of mechanical and systemic failures; however, because humans are the source of design for any given system, clever and motivated individuals will try to defeat any system. Each organization should evaluate the risks posed to a particular nuclear facility based on information related to activist organizations or subversives, disgruntled/disaffected employees, or other information related to the surrounding region. If significant risk from human activity exists, an HRP can help mitigate potential damage.

\section{Why is an HRP important?}

Facilities, organizations, and regulatory agencies have a compelling interest in ensuring that individuals who occupy critical/sensitive positions with access to sensitive information, materials, and/or programs are trustworthy and functioning at their highest level of reliability. An HRP helps to mitigate the internal risk associated with an insider. An insider is any person who has authorized access (either escorted or unescorted) to protected areas. ${ }^{3}$ Insiders can be either managers or employees who could take advantage of their access (i.e., right or opportunity to gain admittance), complemented by their authority (i.e., power or right to enforce obedience) and knowledge of the facility (i.e., awareness or familiarity gained by training or experience), to bypass dedicated physical protection elements or other provisions such as safety, nuclear material control and accountancy, and operating measures and procedures. Motivations of insiders are varied and can range from ideology, revenge, ego, sabotage, or financial need to being forced through coercion by outside elements or even by family members. ${ }^{4}$

Reliability has a logical and direct relationship to trustworthiness because an organization is placing trust in its employees to conduct themselves in a secure, safe, and dependable manner. An HRP provides organizations with a process to help ensure that the highest quality employees are retained in these critical/sensitive positions.

\section{How does an HRP work?}

An HRP uses the initial evaluation for employment (described as follows) to establish the qualifications for being eligible for a sensitive position (one that allows access to sensitive materials, information, or areas). Qualifications for being employed in a position that allows access to sensitive materials are determined by the organization or country. 


\section{INITIAL EVALUATION}

An initial evaluation of a potential employee is the first official check am employer uses to determine if the individual is suited and qualified for employment. Negative issues that cannot be mitigated during this initial evaluation will likely result in a decision not to hire an individual. It is a process to ensure accurate, timely, and equitable determination of an individual's eligibility for access to classified information and materials.

The initial evaluation should include the following components.

- Background Check - A background check consists of gathering information and evaluating an individual through a personal interview and interviews with business associates, friends, and neighbors. These interviews focus on one's character, general reputation, personality traits, and mode of living.

- Initial Drug Test - In the US model, before an individual can be considered for an HRP position, he or she must successfully complete an initial drug screening test. If the drug screening test is positive, a second aliquot (portion) of the sample is sent to a certified narcotic confirmation laboratory to verify and confirm that the test is accurate and results appropriate. A confirmed positive drug test eliminates the individual for further consideration. Drug addiction is a growing problem worldwide and can significantly affect employee performance and safety and has direct security implications. Random alcohol testing may also be conducted on individuals. Each employer must consider whether these test results are potential issues (generically called substance testing) and whether to include them in its program.

- Arrest Check - A check with law enforcement will be conducted to determine if the individual has ever been arrested and, if so, what the charges were (e.g., embezzlement, check fraud, or theft). Engaging in risky/illegal behavior could disqualify an individual from employment.

- Credit Check - A credit check assesses an individual's past borrowing and repaying tendencies, including late payment(s) and indebtedness (how much one owes and to whom). A bad credit report (or owing large sums of money that the applicant has no means to pay) can indicate a potential for blackmail. This is a trait that is not tolerated and could disqualify an individual from employment.

- Education Verification - Checking education background verifies that an individual has attended and graduated from an educational institution (i.e., professional qualifications). Each position includes a list of the job responsibilities and the educational requirements needed to successfully serve in that position.

- Work History Verification - This check validates past employment and time frames (from/to dates) of employment and reveals any work-related performance issues (e.g., disciplinary issues or termination from employment).

- Security Orientation - If hired, this orientation should include training on security requirements and procedures, proper safeguarding of classified information or material, and threat potential and explanation of the individual's obligations and responsibilities. 


\section{CONTINUOUS EVALUATION}

Once an employee has been accepted into a position that requires access to sensitive materials, areas, or information, he or she is then subject to continuous evaluation. Any or all of the Initial Evaluation checks/tests/verifications will be re-administered. Figure 1 shows the Continuous Evaluation process, the generic form of which is called a Structured Trusted Employee Program (STEP). The STEP was developed by the Oak Ridge National Laboratory (ORNL) Center for Human Reliability Safety and Security Studies $\left(\mathrm{CHRS}^{3}\right)$ to be a more generic form of HRP or PRP. Each program has the same set of goals however and allows great flexibility regarding the degree to which an organization implements its program. During an introductory class (Appendix A), many organizations realized that they already had many of the program elements in place but that they were not organized into a stand-alone program. Such organizations may experience a much faster and more effective implementation of their HRP, PRP, or STEP.

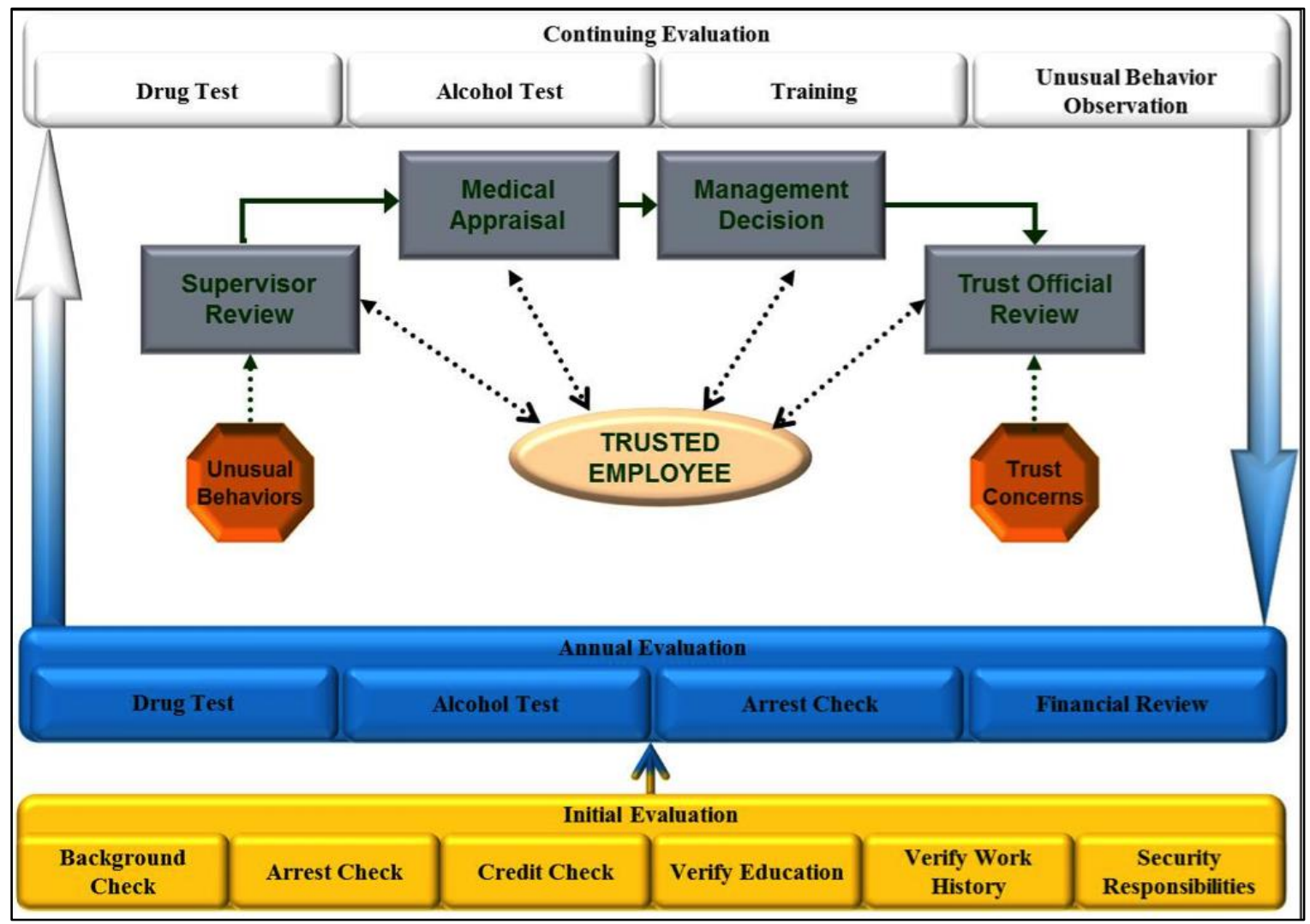

Fig. 1. STEP.

In addition to the Initial Evaluation, the Continuous Evaluation process should include the following components.

- Unusual Behavior - Supervisors, workers, and managers should be trained to identify unusual behavior, the causes of such behavior [e.g., stress, substance (drugs and alcohol) abuse, or medical problems], and ways to distinguish acceptable from unacceptable forms of unusual behavior. All employees should be provided with tools for making accurate observations and following appropriate reporting procedures. With this training in place, managers, supervisors, and staff will be able to effectively monitor their coworkers and alert the proper authorities if questionable behavior is observed. 
- Supervisory Review - Supervisory reviews are required every 12 months regarding the suitability of employees to continue performing HRP work in a safe and reliable manner and thus remain $H R P$ Certified Individuals (Appendix B). Supervisors are trained to evaluate the behaviors and performance of their employees in order to identify security or safety concerns (e.g., failure to comply with work directives, violation of security procedures).

- Medical Appraisal - Employees must undergo an evaluation of their health status and health risk factors, including a medical history review, job task analysis (Appendix C), physical examination, laboratory tests, and psychological and psychiatric evaluations. A nationally normed psychological or psychiatric inventory screening instrument should be considered for HRP-certified positions.

- Management Decision - A designated organizational senior manager evaluates the individual's Supervisory Review and Medical Appraisal, in addition to personnel records related to any security or safety concerns (e.g., garnishment of wages, substance testing). The Management Decision is the first level of approval needed to remain in the HRP; at this step, the senior manager makes the recommendation whether or not to recommend the individual to the Certifying Official.

- Official Review - The Certifying Official (trust official) acts as the final reviewer of all information gathered through the Continuous Evaluation process, and he/she makes the final determination on whether to certify or decertify. This Certifying Official can be part of the facility senior staff (if proper objectivity can be maintained) or part of the government-regulating body, depending on the country's determination.

- Training - HRP-Certified Individuals must complete both initial and annual training requirements, which include understanding the need for an HRP, insider risks, nuclear security awareness, and the their responsibilities. The importance of competently trained staff cannot be overstated. Properly trained employees will have better attitudes toward the security and safety of the facility.

Given the complexity of a nuclear facility, a large number of individuals are granted unescorted access on a daily basis. An insider who has access to and understands the inner workings of the facility presents a serious risk because he/she may have unescorted access to multiple sensitive areas. Possible insider threats and ways to mitigate risk should be considered when identifying critical/sensitive positions. One such way is to develop an HRP, which incorporates security elements that indicate if an individual is unreliable or untrustworthy. Careful identification and monitoring of critical/sensitive positions can reduce the risk from the insider threat.

Careful consideration must be given to those individuals who are granted access to sensitive areas and knowledge of the operations and possible weaknesses of a facility. An effective HRP can help mitigate the insider threat through a Continuous Evaluation process. 


\section{COMPONENTS AND DEVELOPMENT OF AN HRP}

The following portion of this implementation guide provides specific guidance regarding suggested components as well as steps for development of an HRP. Programs may vary because each HRP must be tailored to a particular facility or program to suit its specific circumstances, values, and operational norms. An understanding of the national or governmental regulations/legislations that affect the facility or program is necessary to develop an HRP with the proper security and safety measures in place.

Serious consideration should be given to deciding whether to implement an HRP. Generally, the process begins with subject matters experts (SMEs), who give an overview of the HRP, followed by a more in-depth workshop over about 3 days. The workshop provides detailed information on the technical elements of a reliability program (Appendix A). After the workshop, the facility or organization will have enough information to make an informed decision about pursuing a reliability program.

A team of individuals charged with developing an organization's HRP attend a workshop in which they identify possible elements that should be considered for inclusion in their program (e.g., supervisory review, medical assessments, substance testing). They then develop their program's scope, purpose, and process. If these individuals attend the initial workshop, no additional training is needed; however, a working orientation session with SMEs upon the team's initial formation is advisable. The scope of the program is then submitted to upper management.

Members of the team are also considered HRP Candidates and become HRP Certified once the HRP is initiated. This team is charged with identifying critical/sensitive positions (if needed) and program elements that require HRP certification and the training required for individuals in those positions to become HRP Certified. In addition, individuals administering the HRP (e.g., random alcohol testing, drug testing, and associated procedures) and general requirements for HRP certification are considered to be HRP Candidates and become HRP Certified once the HRP is initiated.

Once the HRP structure is approved, the team develops and documents the organization's policies, procedures, and other required program requirements. This process also establishes the position of the team within the organization.

Upper management then reviews and approves the team's proposed plans and documents, ensuring that they are in accordance with national and state legislation and regulations. Management acceptance and approval are critical to the success of an HRP.

An implementation strategy document prepared by the team is submitted to upper management for approval. This document should include, at a minimum, the following information.

1. Roles and Responsibilities - Identify individuals/units empowered to ensure that the approved HRP elements are in place; approve appropriate policies and procedures; and clearly identify and define organizational relationships.

2. HRP Candidate Positions (if applicable) - Clearly identify critical/sensitive positions (HRP designated) with justifications; develop a detailed process for nominating new positions and deleting existing positions; and accompany all identified positions with a job task analysis.

Training - Provide required initial and annual (refresher) training and schedules (including subject material to be covered). The training process must include those employees in HRP-designated positions (HRP certified) as well as supervisors and managers (particularly those responsible for HRP designated positions) with the knowledge and requirements of the HRP. The program elements may 
be tailored to accommodate group differences and training needs but must include the objectives of the HRP, individual responsibilities, the Continuous Evaluation process, and the benefits of the program. Training can be provided by SMEs or individuals trained by the SMEs at the organization. Trainers must have a strong understanding of the subject material they are to teach. The team also develops a projected start-up schedule for program initiation.

During implementation, policies and procedures are defined and standard operating procedures (SOPs) are written. A parallel document identifies the criteria used to assess the program's progress and appropriate corrective actions that might be taken. Based on the organization's HRP elements, some evaluation criteria should be formalized, and a schedule for this assessment should be documented.

The corrective action matrix is given a specific review schedule and can be changed or modified based on data presented to senior management.

\section{HRP Documentation}

All documentation related to employees' participation in the HRP should be maintained in a separate file with access to the information given on a need-to-know basis. HRP documentation should include, at a minimum, the following records:

1. individuals identified for inclusion in the program and documentation as to when they completed their initial and refresher HRP orientation;

2. signatures on appropriate organization-specific releases, acknowledgments, and waivers indicating an individuals' agreements to participate; and

3. additional HRP information (e.g., security infractions, positive substance tests, and recommendations maintained in accordance with national regulations, organization policies, and implementing regulations).

These files should be maintained as long as an individual is employed by the organization (both while active and inactive in the HRP). Once an individual is no longer employed by the organization, the records should be stored in a secure location for at least 6 months before being destroyed (in compliance with national regulations as well as organization policies and procedures).

\section{HRP Measures of Effectiveness/Evaluation and Corrective Action}

To determine the HRP's effectiveness and associated costs, an organizational metric process needs to be incorporated into the program by an identified unit or individual. The organization must determine whether the program elements are meeting the overall HRP mission.

These measurements provide decision makers with data directly related to program effectiveness. The following is an example using data related to a drug testing element.

- If the data show a decrease in the number of employees with confirmed (screened and confirmed by a certified narcotic confirmation laboratory method as previously mentioned) positive tests for drugs over a given period, then the data demonstrate the effectiveness of this element in improving the reliability of the HRP. A decrease indicates that individuals in critical/sensitive positions are more reliable (i.e., they are not impaired by drugs) and thus are more trustworthy (i.e., they adhere to policies and SOPs as well as support the security and safety culture of the organization/facility). 
- If the data show an increase or steady state of employees with confirmed positive tests for drugs, then the security and safety of the organization/facility are at risk, and corrective action is required.

A Continuous Evaluation process allows senior management to gauge the security and safety culture not only within the organization but also in the context of the broader level of national security. Through a multi-tiered evaluation system, situations and conditions can be identified early that may not be seen routinely. The HRP incorporates many elements to help protect nuclear security and is a powerful and important tool within any nuclear or security organization. HRP depends on reliable and trustworthy employees at all levels of the organization to maintain a robust nuclear security program.

\section{REFERENCES}

1. Based on the model of the US Department of Energy HRP as provided in the US Code of Federal Regulations, Title 10, Part 712 (10 CFR 712).

2. Coates C.W. and G.R. Eisele, Roadmap to a Sustainable Structured Trusted Employee Program (STEP), ORNL/TM-2013/303, August 2013, http://info.ornl.gov/sites/publications/files/Pub45049.pdf

3. IAEA, Nuclear Security Series No. 13, "Nuclear Security Recommendations on Physical Protection of Nuclear Material and Nuclear Facilities" (INFCIRC/225/Revision 5), 2011, http://wwwpub.iaea.org/MTCD/publications/PDF/Pub1481_web.pdf

4. IAEA, Nuclear Security Series No. 8, "Preventive and Protective Measures against Insider Threats, Implementing Guide,” 2008, http://www-pub.iaea.org/MTCD/publications/PDF/pub1359_web.pdf 


\section{APPENDIX A. AN EXAMPLE OF A WORKSHOP AGENDA}

Workshop on Establishing a Human Reliability Program

\section{Day 1 (Main Group)}

$8: 30$

Welcome and Introductions

Host Organization(s)

Technical Implementers (ORNL)

\section{Note: the length of time for a workshop is flexible and dependent on the} organization's requirements

9:00 Introduction to a Human Reliability Programs (HRP)

10:15 Break

10:30 HRP Implementation and Sustainability

This session gives an overview at the top level of the general problem and the need for trusted employees.

12:00 Lunch/Free Time

13:00 Supervisor Reviews

This session gives an overview of the importance of the supervisor in an HRP as the front line of assessment of the employees.

14:15 Break

14:30 Medical Reviews and Psychological Assessments

This session gives an overview of medical criteria for evaluations and of the psychological evaluation process as it relates to reliability as a part of the overall medical evaluation.

15:45 Day 1 Concludes

Day 2 (Main Group)

9:00 Management Reviews

This session covers the organizational requirements of a management review that enables a management official to recommend the continuing certification of an HRP individual.

9:45 Certifying Official Reviews

This session covers the Certifying Official's final review of all HRP information and how to make a determination based on that review for initial and continuing HRP certification.

10:15 Break

10:30 Critical Positions (if applicable)

This session explains how to determine which employees should be covered by HRP.

11:15 Unusual Behavior Observation

This session covers behavioral observation, guidelines for unusual behavior, and what to do when unusual behavior occurs. 
12:00 Lunch/Free Time

13:00 Unusual Behavior Observation (Continued)

14:15 Break

14:30 Unusual Behavior Observation (Continued)

15:45 Day 2 Concludes

\section{Day 3 (Main Group)}

9:00 US Security Evaluation Criteria Example for Pre-hired and Post-hired Staff

This session includes discussion of how security evaluation criteria may relate to the workshop audience.

10:15 Break

10:30 Disgruntled Employee Identification and Employee Satisfaction

This session gives an overview of what constitutes a disgruntled employee and the danger he or she can represent to an organization or facility.

11:15 Insider Threat

This session gives an overview of different types of insiders and insider capabilities and includes case study examples.

12:00 Lunch/Free Time; End of Day for Main Group Participants

\section{Briefing for Managers and Decision Makers on the HRP}

13:00 Introduction to HRP

This session provides an overview of the entire workshop for senior managers.

15:45 Discussion Period

Day 4 (Main Group optional)

9:00 Review of the Main Points of Days 1, 2, and 3

9:30 Case Studies

This session includes examples of insider events from a number of sources to illustrate possible threats.

10:30 Facility Example

This session relates the HRP process to the host facility and involves facility employees in an exercise.

11:15 Break

11:30 Worksheet on Training Needs

This breakout session allows participants to develop multiple program objectives and suggest what future training would benefit the staff facility.

12:45 Group Workshop Concludes

Certificates of Completion are presented, and feedback from participants is requested. 


\section{APPENDIX B. GLOSSARY OF TERMS}

Certification - the formal action the HRP Certifying Official takes that permits an individual to perform HRP duties after it is determined the individual meets the requirements for certification

HRP Administrator - the individuals who oversee the HRP and ensure that program requirements are fulfilled

HRP candidate — an individual being considered for assignment to a position requiring access to sensitive materials, facilities, and programs

HRP-certified individual—an individual who has successfully completed the HRP certification requirements

Human Reliability Program (HRP) — an enhanced security reliability program designed to ensure that individuals in positions requiring access to certain materials, facilities, and programs meet the highest standards of reliability as well as physical and mental suitability

Impaired or impairment - a decrease in the functional capacity of a person that is caused by a physical, mental, emotional, or behavioral disorder or substance abuse

Incident - an unplanned, undesired event that interrupts the completion of an activity and that may include property damage or injury

Insider - any person who has authorized access (either escorted or unescorted) to protected areas

Insider threat - a threat to an organization that comes from people within the organization (such as employees, former employees, contractors, or business associates) who have inside information concerning the organization's security practices, data, and computer systems

Job task analysis - the formal process of defining the requirements of a position and identifying the knowledge, skills, and abilities necessary to effectively perform the duties of the position

Limited access area - designated area containing a nuclear facility and nuclear material to which access is limited and controlled for physical protection purposes

Medical assessment - an evaluation of an HRP candidate or HRP-certified individual's present health status and health risk factors by means of

1. medical history review,

2. job task analysis,

3. physical examination,

4. laboratory tests and measurements, and

5. psychological and psychiatric evaluations.

Nuclear security - the prevention and detection of, as well as the response to, theft, sabotage, unauthorized access, illegal transfer, or other malicious acts involving nuclear material, radioactive substances, or their associated facilities

Nuclear security culture - the assembly of characteristics, attitudes, and behavior of individuals, organizations, and institutions that serves as a means to support and enhance nuclear security

Protected Area-Area inside a limited access area containing nuclear material and/or sabotage targets surrounded by a physical barrier with additional physical protection measures 
Random alcohol testing - the unscheduled, unannounced alcohol testing of randomly selected employees by a process designed to ensure that selections are made in a nondiscriminatory manner

Random drug testing - the unscheduled, unannounced drug testing of randomly selected employees by a process designed to ensure that selections are made in a nondiscriminatory manner

Recertification - the formal action the HRP Certifying Official takes annually (with no more than 12 months between recertifications) permitting an employee to remain in the HRP and perform HRP duties

Reliability - an individual's ability to adhere to security and safety rules and regulations

Safety concern - any condition, practice, or violation that causes a substantial probability of physical harm, property loss, and/or environmental impact

Security concern - the presence of information regarding an individual applying for or holding an HRP position that may be considered derogatory under the country's security criteria

Sensitive position - a position that has access to sensitive materials or information as determined by the facility or country

Supervisor - the individual who has oversight and organizational responsibility for a person holding an HRP position and whose duties include evaluating the behavior and performance of the HRP-certified individual

Unsafe practice - an action causing a person's unnecessary exposure to a hazard or a human action departing from prescribed hazard controls, job procedures, or practices 


\section{APPENDIX C. JOB TASK ANALYSIS}

\section{Job Task Analysis (JTA) Description}

A job task analysis (JTA) is the formal process of defining the requirements of a position and identifying the knowledge, skills, and abilities necessary to effectively perform the duties of that position.

A job is a collection of interrelated tasks and responsibilities that, when performed successfully, results in a desired output of services, for example, materials or product packaged, customer accounts serviced, students taught. Jobs are given titles, such as material handler, teacher, and computer programmer.

A job description is a listing of the tasks and responsibilities that must be accomplished successfully by the person who performs the job.

Jobs are accomplished by the performance of linked tasks. A task is a discrete, measurable unit of work designed to contribute a specified end result to the accomplishment of a job. It has an identifiable beginning and end; it is a measurable component of the duties and responsibilities of a specific job. Tasks may be primarily physical (e.g., lifting), cognitive (e.g., data analysis), or a combination of the two (e.g., security protection).

Analysis of each task involved with a job determines the physical and mental attributes needed by the person who performs the task in order to successfully accomplish the defined job. A job task analysis analyzes elements of the job without regard to the person performing the job.

The analysis of job tasks typically involves two primary categories of requirements: physical and cognitive. The analysis is accomplished through a review of job descriptions, observations, analyses of digital photos or video taken of a sample of the job task cycle(s), measurements of forces, weights, postures, repetitions, and duration of demands. The task analysis is a systematic breakdown of a task into its sub-elements, specifically including a detailed task description of both manual and mental activities, task and element durations, task frequency, task allocation, task complexity, environmental conditions, and any other factors involved in or required to perform a given task.

Physical job tasks frequently are described using such terms as

- cycle frequency and repetitions,

- strength,

- posture,

- complex motions,

- demand duration,

- metabolic demands (peak and range),

- agility,

- vision, visual acuity, and

- hearing threshold.

Cognitive tasks are usually described in terms of skills, knowledge, and attitude requirements. Cognitive task analysis concentrates on the critical decisions and cognitive processes needed for successful outcomes. Terms used to describe cognitive tasks tend to be more complex and job or position specific than those used to describe physical tasks, such as 
- analyze,

- problem solve,

- comprehend,

- calculate,

- forecast,

- manage,

- mentor,

- design,

- administer, and

- perceive.

\section{Role of the JTA in the Human Reliability Program}

A job description tells what is done. The JTA describes how each task is accomplished, the environmental conditions in the area where the task is performed, and the physical and cognitive skills needed to successfully perform each task. The JTA provides an objective tool to determine if individuals are suited for a specific job, provides matrices for performance evaluations, and serves as an objective standard for the determination of numerous return-to-work decisions, including questions of qualification versus disqualification. The JTA defines the required set of standards for an individual performing a given job. Without a valid JTA, decisions related to initial hiring, performance evaluations, and return-to-work decisions become subjective, rather than objective. 
